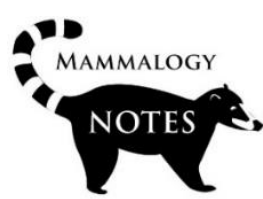

\title{
Registro notable de armadillo de cola desnuda, Cabassous centralis (Cingulata: Chlamyphoridae), en el estado de Chiapas, México
}

\author{
Saúl Sánchez-Soto*iD, Carlos M. Ramírez-Arenas² (D), Nabor Esteban-Guzmán³ (iD, \\ Wendy Susana Sánchez-Gómez ${ }^{4}$ (D) \\ 1 Colegio de Postgraduados, Campus Tabasco. Río Seco y Montaña 2da. Sección, C.P. 86402, Huimanguillo, Tabasco, México. \\ 2 Bajo Tulijá, C.P. 86746, Macuspana, Tabasco, México. \\ 3 Buena Vista (Apasco), C.P. 86745, Macuspana, Tabasco, México. \\ 4 Universidad Autónoma de Yucatán, Campus de Ciencias Biológicas y Agropecuarias, C.P. 97315, Mérida, Yucatán, México. \\ *Correspondencia: sssoto@colpos.mx
}

\section{Resumen}

El 19 de abril de 2020 se observó y fotografió un individuo de Cabassous centralis capturado por trabajadores de campo en un fragmento de vegetación secundaria en el ejido Jilumil, municipio de Salto de Agua, en el norte de Chiapas, México. Este registro es actualmente el más septentrional en la distribución conocida de esta especie, el cual se ubica a $18 \mathrm{~km}$ del registro más cercano publicado anteriormente.

Palabras clave: armadillo de cola desnuda, México, norte de Chiapas, registro.

\section{Abstract}

On April 19, 2020, an individual of Cabassous centralis captured by field workers in a fragment of secondary vegetation in the Jilumil ejido, Salto de Agua municipality, in northern Chiapas, Mexico, was observed and photographed. This record is currently the northernmost in the distribution of this species, which is located $18 \mathrm{~km}$ of the closest previously published record.

Key words: Northern naked-tailed armadillo, Mexico, northern Chiapas, record.

En México habitan dos especies de armadillos, el armadillo común (Dasypus novemcinctus) que se distribuye en gran parte del país, y el armadillo de cola desnuda (Cabassous centralis) cuya distribución se limita al estado de Chiapas (Cuarón 2005). Este último es una especie solitaria, nocturna y rara, la cual se alimenta principalmente de hormigas y termitas, aunque su historia natural es poco conocida (Aranda \& March 1987; Cuarón et al. 1989; Eisenberg 1989; Cuarón 2005; Hayssen et al. 2012). Es un armadillo pequeño que pesa entre 2.5 y $3.5 \mathrm{~kg}$; se distingue por su cabeza y hocico cortos y anchos, caparazón con 12 o 13 bandas y con escamas óseas de forma casi cuadrangular, cola corta y desprovista de placas epidérmicas y garra del tercer dedo de las patas anteriores relativamente grande (Aranda \& March 1987; Cuarón 2005). Su distribución conocida comprende desde el norte de Chiapas, México, hasta Venezuela y Ecuador, desde el nivel del mar hasta los $3.018 \mathrm{msnm}$ (Eisenberg 1989; Hayssen et al. 2012; Juárez-López et al. 2017; Santos et al. 2019). Se ha registrado en diferentes tipos de bosque tropical, así como en vegetación secundaria, 
sabanas, pastizales y agroecosistemas (Eisenberg 1989; Cuarón 2005; Cruz-Rodríguez et al. 2011; Hayssen et al. 2012; Sánchez-Brenes \& Monge 2019).

En México se registró por primera vez en 1989 en la región conocida como Selva Lacandona con base en un individuo vivo confiscado en octubre de 1986 procedente de San Javier, localidad cercana al sitio arqueológico de Bonampak, en una zona adyacente a la parte norte de la Reserva de la Biósfera Montes Azules (REBIMA), en el municipio de Ocosingo (Cuarón et al. 1989) (Figura 1). Desde entonces, se han obtenido aproximadamente 60 registros de la especie en diferentes puntos del estado, principalmente alrededor de la REBIMA y sitios adyacentes, cerca de los límites con Guatemala (Naranjo et al. 2004; González-Zamora et al. 2011; Garmendia et al. 2013; Figueroa-De León et al. 2016; JuárezLópez et al. 2017; Santos et al. 2019) (Figura 1). Cuatro de estos registros sobresalen porque se ubican en el norte del estado, a distancias considerables del sitio donde este mamífero se registró por primera vez en el país (Juárez-López et al. 2017; Santos et al. 2019) (Figura 1). En la presente nota se presenta un nuevo registro de distribución de $C$. centralis en el norte del Estado de Chiapas.

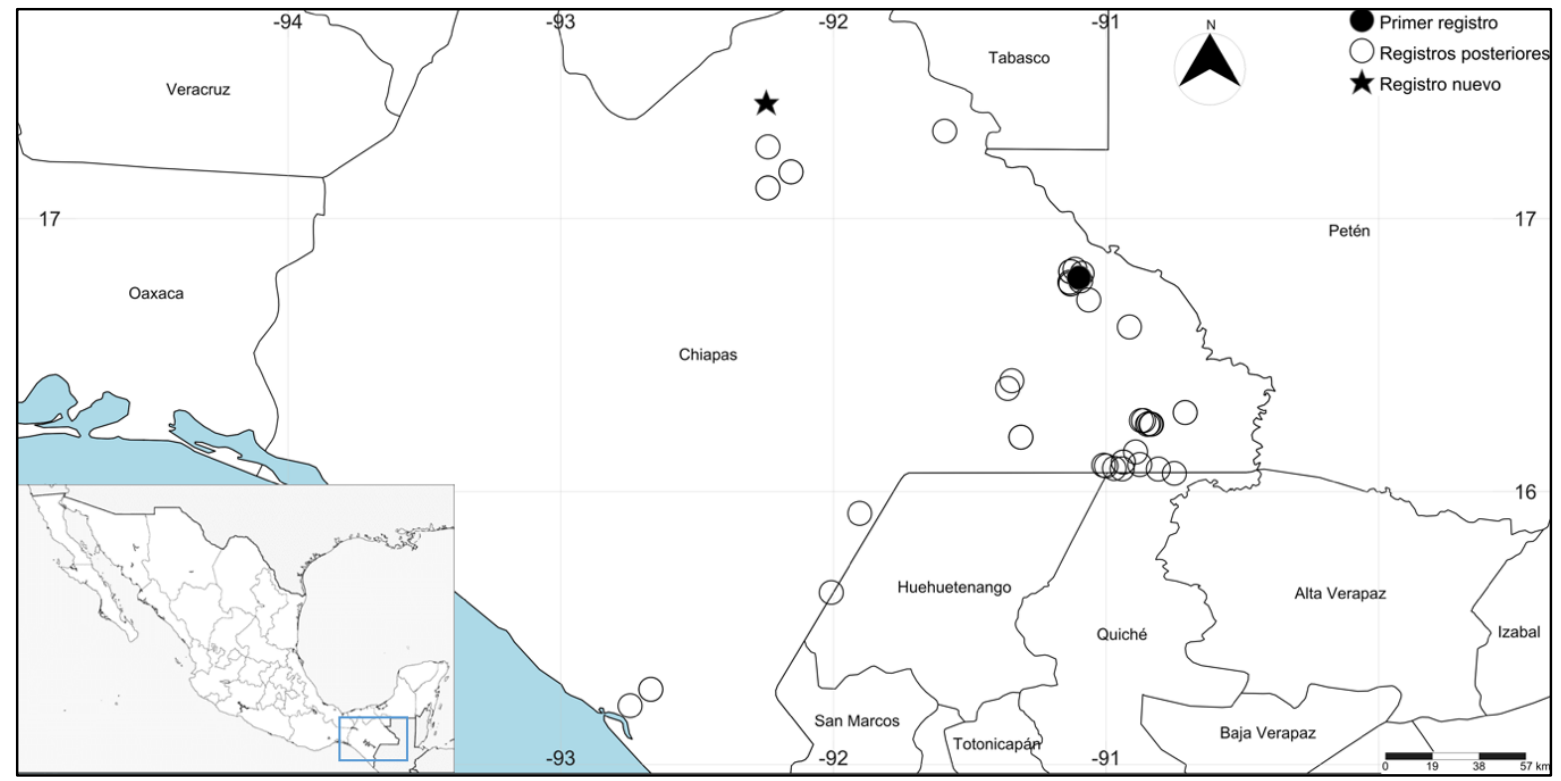

FIGURA 1. Área de estudio con los registros publicados de Cabassous centralis en México, de 1986 a 2020.

El 19 de abril del 2020, cerca de las 13:00 horas, se registró la presencia de un individuo adulto de C. centralis (Figura 2), el cual se encontró en una cavidad en el suelo y posteriormente fue capturado por trabajadores de campo en un fragmento de vegetación secundaria (acahual) en el rancho Los Pastores, en el ejido Jilumil, municipio de Salto de Agua, en el norte de Chiapas, a una altitud de 60 msnm (17.426150, -92.246310 WGS84). Creyendo que se trataba del armadillo común (D. novemcinctus), dichos trabajadores lo extrajeron de la cavidad con ayuda de una pala coa. Debido a que fue herido considerablemente en la parte posterior del cuerpo con esta herramienta, tuvo que ser sacrificado, para ser posteriormente consumido por algunos pobladores.

La vegetación de la zona está compuesta principalmente por pastizales para la cría de ganado vacuno con fragmentos de vegetación secundaria y árboles dispersos. La fisiografia corresponde a la Llanura Costera del Golfo Sur. El clima es cálido húmedo con 
lluvias todo el año; la variación isotermal es de 26 a $28^{\circ}$ C y la variación pluvial de 3.500 a $4.000 \mathrm{~mm}$ al año (INEGI 2017).

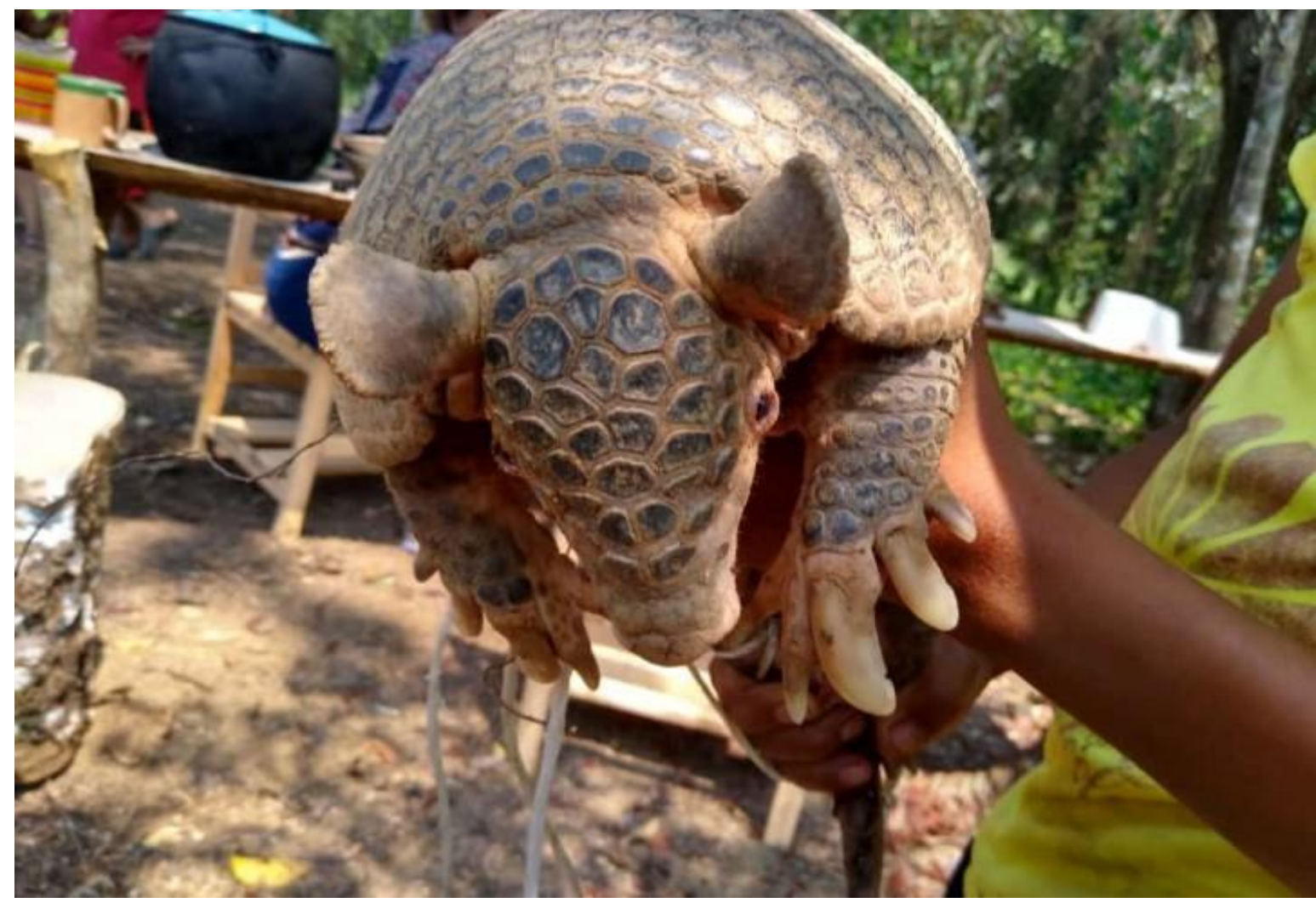

FIGURA 2. Individuo de Cabassous centralis registrado en el ejido Jilumil, Salto de Agua, Chiapas.

Cabassous centralis está catalogado como Datos Insuficientes (DD) a nivel internacional (Tirira et al. 2014) y se encuentra en peligro de extinción para México debido principalmente a la destrucción de su hábitat (SEMARNAT 2010). Sin embargo, la muerte de individuos por atropellamientos y por la cacería debido a la creencia que es una especie venenosa, constituyen otros factores que la hacen vulnerable a la extinción aunada a su rareza, por lo cual los proyectos de educación ambiental son esenciales para ayudar a cambiar la percepción que la población local tiene sobre esta especie (González-Zamora et al. 2011).

El registro descrito es relevante porque su ubicación constituye actualmente el sitio más al norte de la distribución conocida de C. centralis, a $18 \mathrm{~km}$ al norte del registro más cercano publicado anteriormente (Santos et al. 2019), y a $141 \mathrm{~km}$ al noroeste del lugar donde la especie se registró por primera vez en México (Cuarón et al. 1989). Aunque el sitio de registro no se encuentra dentro de un área natural protegida, se sugiere realizar un estudio en la zona con el fin de conocer las amenazas, la densidad poblacional de este mamífero y la importancia que tienen los fragmentos de vegetación secundaria para su supervivencia, de modo que constituya una base para implementar medidas de protección. 


\section{AGRADECIMIENTOS}

A los revisores anónimos por sus valiosas observaciones y aportaciones que mejoraron la presentación del trabajo.

\section{REFERENCIAS}

Aranda M, March I. 1987. Guía de los mamíferos silvestres de Chiapas. 1 edición. Instituto Nacional de Investigaciones sobre Recursos Bióticos. Xalapa, Veracruz, México.

Cruz-Rodríguez CA, Larrotta L, González-Maya JF, Zárrate-Charry D, Cepeda AA, Balaguera-Reina SA, Ange-Jaramillo C, Zamora A, Castaño-Uribe C. 2011. New records for the northern nakedtailed armadillo Cabassous centralis (Cingulata: Dasypodidae) in tropical dry forest of the department of Sucre, Colombian Caribbean. Revista Mexicana de Mastozoología Nueva Época 1:39-45.

Cuarón, A.D., 2005. Cabassous centralis (Miller, 1899), armadillo cola de zorro, in: Ceballos, G., Oliva, G. (Coords.), Fondo de Cultura Económica, y Comisión Nacional para el Conocimiento y Uso de la Biodiversidad, México, D.F., pp. 116-117.

Cuarón AD, March IJ, Rockstroh PM. 1989. A second armadillo (Cabassous centralis) for the faunas of Guatemala and Mexico. Journal of Mammalogy 70:870-871. https://doi.org/10.2307/1381731

Eisenberg JF. 1989. Mammals of the Neotropics: Panamá, Colombia, Venezuela, Guyana, Suriname, French Guiana. The University of Chicago Press. Chicago, USA.

Figueroa-De León A, Naranjo EJ, Santos-Moreno A. 2016. Registros de Cabassous centralis (Cingulata: Dasypodidae) en la Reserva de la Biósfera Montes Azules y sitios aledaños, Chiapas, México. Edentata 17:46-50. https:// doi.org/10.2305/IUCN.CH.2016.EDENTATA-17-1en.

Garmendia A, Arroyo-Rodríguez V, Estrada A, Naranjo EJ, Stoner KE. 2013. Landscape and patch attributes impacting médium- and large-sized terrestrial mammals in a fragmented rain forest. Journal of Tropical Ecology 29:331-344. https://doi.org/10.1017/S0266467413000370

González-Zamora A, Arroyo-Rodríguez V, González-Di Pierro AM, Lombera R, de la Peña-Cuéllar E, Peña-Mondragón JL, Hernández-Ordoñez O, Muench C, Garmendia A, Stoner KE. 2011. The northern naked-tailed armadillo in the Lacandona rainforest, Mexico: new records and potential threats. Revista Mexicana de Biodiversidad 82:581-586.

Hayssen V, Ortega J, Morales-Leyva A, Martínez-Méndez N. 2012. Cabassous centralis (Cingulata: Dasypodidae). Mammalian Species 45:12-17. https:// doi.org/10.1644/898.1.

INEGI. 2017. Anuario estadístico y geográfico de Chiapas 2017. Instituto Nacional de Estadística y Geografía. México.

Juárez-López R, Pérez-López M, Bravata-de la Cruz Y, Jesús-de la Cruz A, Contreras-Moreno FM, Thornton D, Hidalgo-Mihart MG. 2017. Range extensión of the northern naked-tailed armadillo (Cabassous centralis) in southern Mexico. Western North American Naturalist 77:398-403. https:// doi.org/10.3398/064.077.0311

Naranjo EJ, Guerra MM, Bodmer RE, Bolaños JE. 2004. Subsistence hunting by three ethnic groups of the Lacandon Forest, Mexico. Journal of Ethnobiology 24:233-253.

Sánchez-Brenes RJ, Monge J. 2019. El armadillo, Cabassous centralis (Cingulata: Chlamyphoridae) en agroecosistemas con café de Costa Rica. Cuadernos de Investigación UNED 11:436-443. https:// doi.org/10.22458/urj.v11i3.2724. 
Santos PM, Bocchiglieri A, Chiarello AG, Paglia AP, Moreira A, De Souza AC, Abba AM, Paviolo A, Gatica A, Medeiro AZ. 2019. Neotropical Xenarthrans: a data set of occurrence of Xenarthran species in the Neotropics. Ecology 100(7):e02663. https:// dx.doi.org/10.1002/ ecy.2663.

SEMARNAT. 2010. Norma Oficial Mexicana NOM-059- SEMARNAT-2010. Protección ambiental. Especies nativas de México de flora y fauna silvestres. Categorías de riesgo y especificaciones para su inclusión, exclusión o cambio. Lista de especies en riesgo. Diario Oficial, 30 de diciembre de 2010, 2a Sección. México DF. México.

Tirira DG, Díaz-N, J, Superina M, Abba AM. 2014. Cabassous centralis. The IUCN Red List of Threatened Species 2014: e.T3412A47437304. Accessed on 14 June 2020. https://dx.doi.org/10.2305/IUCN.UK.2014-1.RLTS.T3412A47437304.en

Editor: José F. González-Maya

Recibido 2020-05-05

Revisado 2020-06-05

Aceptado 2020-06-07

Publicado 2020-07-21 